P. Malcovati, S. Brigati, F. Francesconi, F. Maloberti, P. Cusinato, A. Baschirotto: "Behavioral modeling of switched-capacitor sigma-delta modulators"; IEEE Transactions on Circuits and Systems I: Fundamental Theory and Applications, Vol. 50, Issue 3, 2003, pp. 352-364.

(C)20xx IEEE. Personal use of this material is permitted. However, permission to reprint/republish this material for advertising or promotional purposes or for creating new collective works for resale or redistribution to servers or lists, or to reuse any copyrighted component of this work in other works must be obtained from the IEEE. 


\title{
Behavioral Modeling of Switched-Capacitor Sigma-Delta Modulators
}

\author{
Piero Malcovati, Member, IEEE, Simona Brigati, Member, IEEE, Fabrizio Francesconi, Member, IEEE, \\ Franco Maloberti, Fellow, IEEE, Paolo Cusinato, and Andrea Baschirotto, Senior Member, IEEE
}

\begin{abstract}
This paper presents a complete set of blocks implemented in the popular MATLAB SIMULINK environment, which allows designers to perform time-domain behavioral simulations of switched-capacitor (SC) sigma-delta $(\Sigma \Delta)$ modulators. The proposed set of blocks takes into account most of the SC $\Sigma \Delta$ modulator nonidealities, such as sampling jitter, $k T / C$ noise, and operational amplifier parameters (white noise, finite dc gain, finite bandwidth, slew rate and saturation voltages). For each block, a description of the considered effect as well as all of the implementative details are provided. The proposed simulation environment is validated by comparing the simulated behavior with the experimental results obtained from two actual circuits, namely a second-order low-pass and a sixth-order bandpass SC $\Sigma \Delta$ modulator.
\end{abstract}

Index Terms-Analog-digital conversion, discrete-time systems, sigma-delta $(\Sigma \Delta)$ modulation.

\section{INTRODUCTION}

$\mathbf{S}$ IGMA-DELTA $(\Sigma \Delta)$ modulators are the most suitable A/D converter topologies for digitizing with high-resolution analog signals characterized by a bandwidth (BW) much smaller than the sampling frequency $\left(f_{s}\right)$. With these architectures, a resolution up to 19-21 bits can be reached using standard IC technologies [2]-[4]. These features make the $\Sigma \Delta$ solutions very attractive for a number of applications. For instance, they have gained increasing popularity in audio applications (where a low-pass signal BW requires a resolution up to $20 \mathrm{bit}$ ), in receivers for communication systems (where the modulated signal can be digitized at the interface with a bandpass $\Sigma \Delta$ modulator), in sensor interface circuits and in measurement systems. Key advantages of $\Sigma \Delta$ modulators are their inherent linearity and their robust analog implementation. Moreover, by trading accuracy with speed, $\Sigma \Delta$ modulators allow high performance to be achieved with low sensitivity to analog component imperfections and without requiring component trimming.

Manuscript received February 5, 2002; revised October 16, 2002. This paper was recommended by Associate Editor O. Feely.

P. Malcovati is with the Department of Electrical Engineering, University of Pavia, 27100 Pavia, Italy (e-mail: piero@ele.unipv.it).

S. Brigati and F. Francesconi are with ACCO Microelettronica S.r.1., 27100

Pavia, Italy (e-mail: s.brigati@acco-ic.it; f.francesconi@acco-ic.it).

F. Maloberti is with the Department of Electronics, University of Pavia, 27100

Pavia, Italy (e-mail: franco@ele.unipv.it).

P. Cusinato is with the Texas Instruments France, 06270 Villeneuve Loubet, France (e-mail: p-cusinato@ti.com).

A. Baschirotto is with the Department of Innovation Engineering, University

of Lecce, 73100 Lecce, Italy (e-mail: andrea.baschirotto@unile.it).

Digital Object Identifier 10.1109/TCSI.2003.808892
$\Sigma \Delta$ modulators can be implemented either with continuous-time or with sampled-data techniques. The most popular approach is based on a sampled-data solution with switchedcapacitor (SC) implementation. In fact, SC $\Sigma \Delta$ modulators can be efficiently realized in standard CMOS technology and included in complete mixed-signal systems without any performance degradation. For this reason, we will focus on the case of SC $\Sigma \Delta$ modulators in this paper.

In the design of a high-performance SC $\Sigma \Delta$ modulator, two main issues have to be addressed by the designers.

1) Which is the best architecture to fulfill the application requirements?

2) For a given architecture, which are the requirements for the building blocks?

In practice, a significant problem in the design of $\Sigma \Delta$ modulators is the estimation of their performance, since they are mixed-signal nonlinear circuits. Due to the inherent nonlinearity of the $\Sigma \Delta$ modulator loop the optimization of the performance has to be carried out with behavioral time-domain simulations. This situation is quite difficult to handle when a high-performance system is considered. Indeed, to satisfy high-performance requirements, accurate simulations of a number of nonidealities and, eventually, the comparison of the performance of different architectures are needed in order to choose the best solution. In addition to this, in the design of high-resolution SC $\Sigma \Delta$ modulators, a large set of parameters, including the performance of the building blocks embedded in the adopted structure, has to be optimized in order to achieve the desired signal-to-noise ratio (SNR) or signal-to-noise and distortion ratio (SNDR). ${ }^{1}$

In principle, various approaches for transient simulation which include device models (such as SPICE), finite-difference equations (such as SWITCAP), custom numerical models (typically in $\mathrm{C}++$ language), etc., are already available. However, in different measures, all of them exhibit some disadvantages. Table I [5] classifies the different tools in terms of three main characteristics: accuracy, speed, and flexibility (intended as modeling capability plus reusability). Moreover, the post-processing algorithms for the evaluation of modulator performances are other qualifying features for the various tools.

SPICE is a conventional electrical simulator and, despite its precision, it is not suitable for the analysis of $\Sigma \Delta$ modulators be-

\footnotetext{
${ }^{1}$ Notice that the two issues mentioned above are strictly correlated because architectures which achieve optimal results with ideal block models are not necessarily the best solution in the presence of building blocks with degraded performances. Therefore, the choice of the architecture and design of the building blocks require typically concurrent optimization processes.
} 
TABLE I

COMPARISON AMONG THE DifFERENT TOOLS FOR THE Simulation of $\Sigma \Delta$ MOdUlator

\begin{tabular}{|c|c|c|c|}
\hline Approach & Accuracy & Speed & Flexibility \\
\hline Device models & (:) & : : & 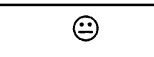 \\
\hline Custom models $(\mathrm{C}++)$ & $\odot$ & (:) & : $:$ \\
\hline Finite-difference equations & $\odot$ & ;) & $\oplus$ \\
\hline Circuit-based macromodel & ;) & : : & (:) \\
\hline Time-domain macromodel & (:) & $\oplus$ & (2) \\
\hline Table-lookup model & 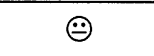 & $\oplus$ & : $:$ \\
\hline Behavioral models & (:) & 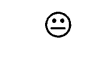 & (:) \\
\hline Proposed solution & (:) & (:) & (-) \\
\hline
\end{tabular}

cause of the extremely long simulation time. Custom models are just suited for a specific structure and cannot be easily adapted to a different modulator topology (especially regarding exotic architectures). This situation is quite difficult to handle when accurate simulations of a number of nonidealities and, eventually, the performance comparison between different architectures are needed.

The circuit-based macro model essentially represents an equivalent circuit build up with a minimum set of passive and active devices already available in electrical simulators like SPICE. Modulator building blocks are described by means of simplified circuits and specifications are used as model parameters. Nonidealities can be introduced in the models. This approach guarantees a good degree of accuracy and reusability, but the speed improvement with respect to device level simulation is poor. Time-domain macro models are based on a set of equations describing the transient behavior of a specific circuit. The specifications of the circuit represent the model parameters. Again this approach is not flexible at all, but allows us to introduce dynamic nonlinearities.

The simulators based on finite-difference equations are programs usually written in $\mathrm{C}$ language that exploit the $z$-domain description of the transfer function of sample-data networks. They can be general-purpose like SWITCAP (or its evolution AWEswit [6]) or especially devoted to oversampled modulators like MIDAS. They achieve an excellent speed of simulation, but the nonidealities modeling capabilities are poor. Moreover, both simulators operate on netlists and offer a not user-friendly human interface.

Table-lookup models [7] use a two-step procedure. First, tables of input and output points are extracted for the $\Sigma \Delta$ modulator sub-blocks by using conventional electrical simulators. Then, the obtained tables are utilized instead of the original circuit for global transients simulations. However, this approach seems not to guarantee high accuracy $(<80 \mathrm{~dB})$ in SNR estimation (static errors only) and tables are not reusable. The speed of this approach depends on the size of the tables.

Further behavioral simulator with a more custom approach like simulators or those reported in [8] and [9], represent a compromise between finite-difference equations simulators and behavioral model simulators achieving intermediate performances.

In this paper, a complete set of blocks to be used in the very popular MATLAB SIMULINK [10] environment is proposed. This toolbox allows us to perform exhaustive time-domain behavioral simulations of $\Sigma \Delta$ modulators. The most significant nonidealities are modeled and building blocks for modeling sampling jitter, $k T / C$ noise, and operational amplifier parameters (white noise, finite dc gain, finite BW, slew rate (SR) and saturation voltages) are proposed.

It is important to point out that the proposed building blocks allow us to achieve a good estimation of the SC $\Sigma \Delta$ modulator performance. The proposed models, however, are not perfect, since they are based on a number of hypothesis which are typically, but not always, verified in $\Sigma \Delta$ modulators. In the development of this particular set of blocks, taking into account the needs of the $\Sigma \Delta$ modulator designers, the basic tradeoff between accuracy and simplicity of the models has been optimized in terms of model simplicity and hence, efficiency.

The proposed behavioral simulation toolbox (available on the worldwide web [1]), together with the utilities distributed in [11], has been used for designing, simulating, and verifying the performance of two actual $\Sigma \Delta$ modulators: a second-order 15-bit low-pass and a sixth-order 12-bit bandpass modulator. The simulated performance of these circuits is compared with experimental results in order to validate the proposed simulation environment.

This paper is organized as follows. In Section II, the major nonidealities of SC $\Sigma \Delta$ modulators are described. The correspondent behavioral model blocks are then presented in Section III- V. Finally, in Section VI, we report the results obtained with the proposed blocks for a second-order low-pass and for a sixth-order bandpass $\Sigma \Delta$ modulator.

\section{II. $\Sigma \Delta$ MOdUlator NonidealitiES}

The block diagram of a first-order SC $\Sigma \Delta$ modulator is shown in Fig. 1. This circuit will be used in this section, to introduce the nonidealities which affect the performance of SC $\Sigma \Delta$ modulators of any order. The modulator consists of an input sampler, a 


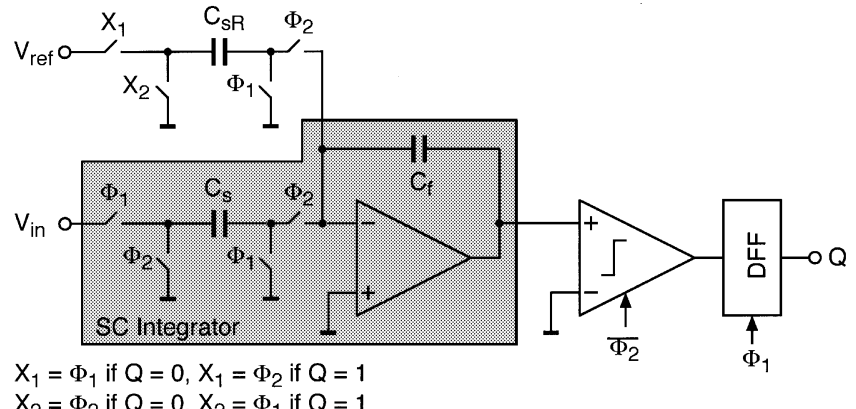

$X_{2}=\Phi_{2}$ if $Q=0, X_{2}=\Phi_{1}$ if $Q=1$

Fig. 1. Schematic of an SC first-order $\Sigma \Delta$ modulator.

SC integrator, a quantizer and a feedback digital-to-analog converter (DAC). The main nonidealities of this circuit which are considered in this paper are the following:

1) clock jitter at the input sampler;

2) switch thermal noise in the SC structure;

3) operational amplifier noise;

4) operational amplifier finite gain;

5) operational amplifier BW;

6) operational amplifier SR;

7) operational amplifier saturation voltages.

The use of the SC technique for the implementation means that all the blocks in a SC $\Sigma \Delta$ modulator are properly synchronized. Using the building blocks presented in the following sections, the simulation of any $\mathrm{SC} \Sigma \Delta$ modulator is possible.

The basic concept of the proposed simulation environment is the evaluation of the output samples in the time domain. The nonidealities listed above produce a deviation of the output samples from their ideal values. The overall performance of the $\Sigma \Delta$ modulator is then evaluated in the frequency domain after proper fast Fourier transform (FFT) [12] of the output samples (see the Appendix).

\section{Clock JitTER}

The operation of an SC circuit depends on complete charge transfers during each of the clock phases [13]. Once the analog signal has been sampled, the SC circuit is a sampled-data system where variations of the clock period have no direct effect on the circuit performance. Therefore, the effect of clock jitter on an SC circuit is completely described by computing its effect on the sampling of the input signal. This also means that the effect of clock jitter on a $\Sigma \Delta$ modulator is independent of the structure or order of the modulator.

Clock jitter results in a nonuniform sampling time sequence, and produces an error which increases the total error power at the quantizer output. The magnitude of this error is a function of both the statistical properties of the jitter and the modulator input signal. The error introduced when a sinusoidal signal $x(t)$ with amplitude $A$ and frequency $f_{\text {in }}$ is sampled at an instant which is in error by an amount $\delta$ is given by

$$
x(t+\delta)-x(t) \approx 2 \pi f_{\text {in }} \delta A \cos \left(2 \pi f_{\text {in }} t\right)=\delta \frac{d}{d t} x(t) .
$$

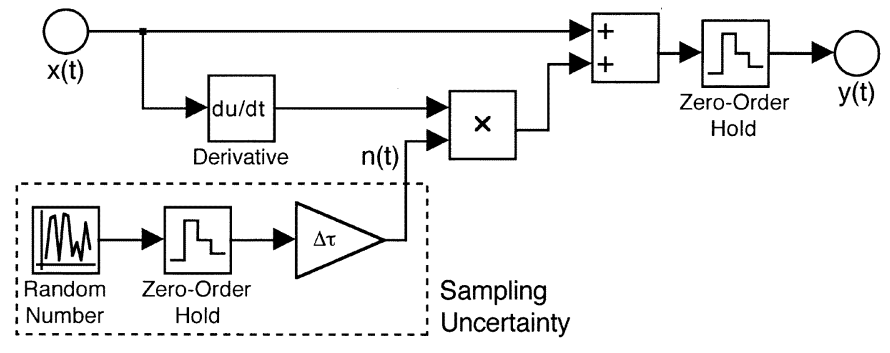

Fig. 2. Modeling a random sampling jitter.

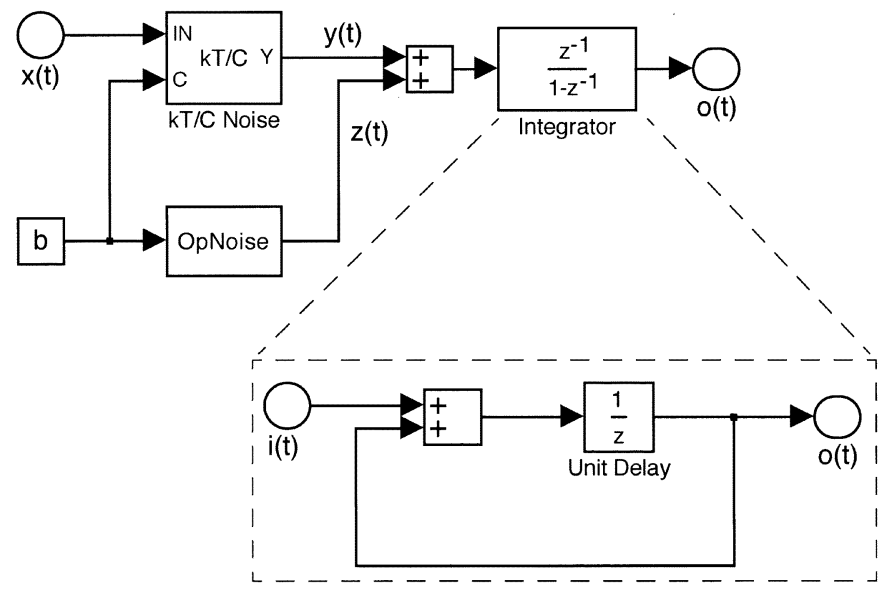

Fig. 3. Model of a "noisy" integrator.

This effect can be simulated at behavioral level by using the model shown in Fig. 2, which implements (1). The input signal $x(t)$ and its derivative $(d u / d t)$ are continuous-time signals. They are sampled with sampling period $T_{s}$ by a zero-order hold. In the model, we assumed that the sampling uncertainty $\delta$ is a Gaussian random process $n(t)$ with standard deviation $\Delta \tau$. The signal $n(t)$ is implemented starting from a sequence of random numbers with Gaussian distribution, zero mean, and unity standard deviation (available in SIMULINK). Other possible distributions can be considered and consequently implemented in this block. Whether oversampling is helpful in reducing the error introduced by the jitter depends on the nature of the jitter. Since we assume the jitter white, the resultant error has uniform power-spectral density (PSD) from 0 to $f_{s} / 2$, with a total power of $\left(2 \pi f_{\text {in }} \Delta \tau A\right)^{2} / 2$. In this case, the total error power will be reduced by the oversampling ratio [14].

\section{Thermal AND OPERATIONAL AMPLIFIER NOISE}

The most important noise sources affecting the operation of an SC $\Sigma \Delta$ modulator are the thermal noise associated to the sampling switches and the intrinsic noise of the operational amplifiers.

These effects can be successfully simulated at the behavioral level by using the model of a "noisy" integrator shown in Fig. 3, which represents the SC integrator shown in Fig. 1 (a similar model can be used also for resonators). The $z$-domain transfer function of this integrator $H_{I}(z)$ is given by

$$
H_{I}(z)=\frac{C_{s}}{C_{f}} \frac{z^{-1}}{1-z^{-1}}=b \frac{z^{-1}}{1-z^{-1}}
$$




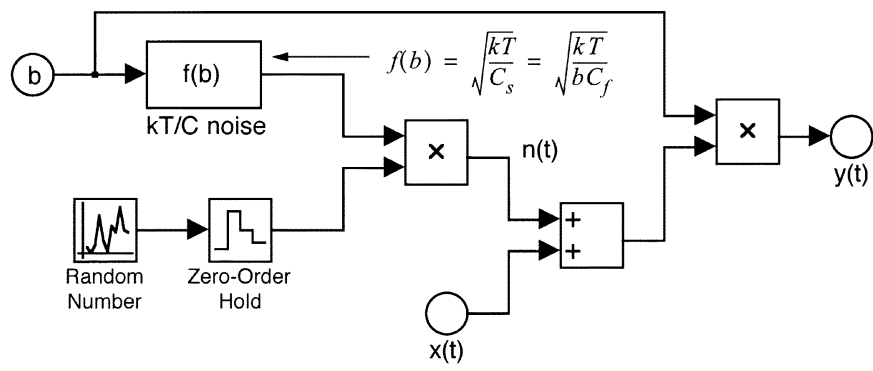

Fig. 4. Modeling switches thermal noise ( $k T / C$ block).

where the variable $b=C_{s} / C_{f}$ represents the coefficient of the integrator. The input signal $x(t)$ is multiplied by $b$ in the $k T / C$ block.

Each noise source and its relevant model will be described in the following paragraphs.

\section{A. Switches Thermal Noise}

Thermal noise is caused by the random fluctuation of carriers due to thermal energy and is present even at equilibrium. Thermal noise has a white spectrum and wide band, limited only by the time constant of the switched capacitors or the BW of the operational amplifiers. Referring to the SC first-order $\Sigma \Delta$ modulator shown in Fig. 1, the sampling capacitor $C_{s}$ is in series with a switch, with finite resistance $R_{\mathrm{on}}$, that periodically opens, thus sampling a noise voltage onto $C_{s}$. The total noise power can be found evaluating the integral [5]

$$
e_{T}^{2}=\int_{0}^{\infty} \frac{4 k T R_{\mathrm{on}}}{1+\left(2 \pi f R_{\mathrm{on}} C_{s}\right)^{2}} d f=\frac{k T}{C_{s}}
$$

where $k$ is the Boltzmann's constant, $T$ the absolute temperature, and $4 k T R_{\text {on }}$ the noise PSD associated with the switch on-resistance. The switch thermal noise voltage $e_{T}$ (usually called $k T / C$ noise) is then superimposed to the input voltage $x(t)$ leading to

$$
\begin{aligned}
y(t) & =\left[x(t)+e_{T}(t)\right] b \\
& =\left[x(t)+\sqrt{\frac{k T}{C_{s}}} n(t)\right] b \\
& =\left[x(t)+\sqrt{\frac{k T}{b C_{f}}} n(t)\right] b
\end{aligned}
$$

where $n(t)$ denotes a Gaussian random process with unity standard deviation, while $b=C_{s} / C_{f}$ is the coefficient of the integrator. Equation (4) is implemented by the model shown in Fig. 4.

The integrators or resonators of an SC $\Sigma \Delta$ modulator may include more than one SC input branch, each contributing to the total noise power. For example, in the $\Sigma \Delta$ modulator shown in Fig. 1, there are two input branches, one carrying the signal and the other providing the feedback from the modulator output. Each branch has to be modeled with a separate $k T / C$ noise block, including the proper coefficient $b$ (different coefficients can be used in the different branches).

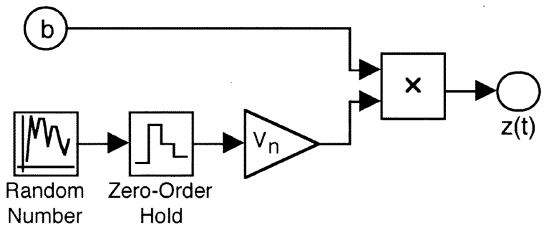

Fig. 5. Operational amplifier noise model (OpNoise block).

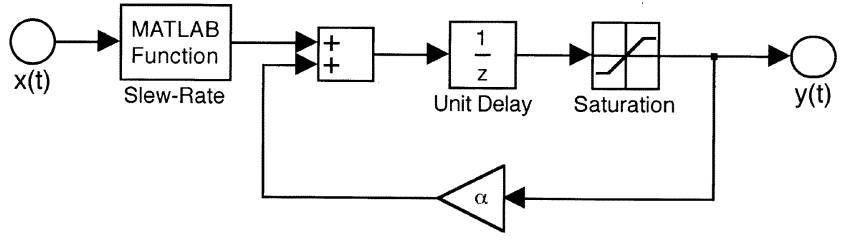

Fig. 6. Real integrator model.

\section{B. Operational Amplifier Noise}

Fig. 5 shows the model used to simulate the effect of the operational amplifier noise [15]. Here, $V_{n}$ represents the total rms noise voltage of the operational amplifier referred to the integrator (or resonator) input. In this model we considered only thermal noise, while flicker $(1 / f)$ noise and dc offset are neglected. Indeed, in low-pass $\Sigma \Delta$ modulators, flicker noise and dc offset are typically canceled by means of auto-zero, correlated double sampling, or chopper stabilization techniques, while they are not important in bandpass architectures. The noise power $V_{n}^{2}$ can be evaluated through a transistor-level noise simulation of the complete integrator in the proper clock phase, including feedback, sampling and load capacitors (clock phase $\Phi_{2}$ in Fig. 1). The resulting output referred noise PSD has to be integrated over the whole frequency spectrum, eventually taking into account the degradation of the thermal noise PSD introduced by the auto-zero or correlated double sampling techniques [16], and then divided by $b^{2}$ in order to refer the obtained noise power to the integrator input.

In this model, we consider only the contribution of the sampled noise, assuming that no continuous-time paths are present across the $\Sigma \Delta$ modulator (this is typically the case in SC $\Sigma \Delta$ modulators due to the presence of a latched quantizer).

\section{OPERATIONAL AMPLIFIER NONIDEALITIES}

The behavioral model of an ideal integrator with unity coefficient is shown in the inset of Fig. 3. Its transfer function is

$$
H(z)=\frac{z^{-1}}{1-z^{-1}} .
$$

Analog circuit implementations of the integrator or resonator deviate from this ideal behavior due to several nonideal effects. One of the major causes of performance degradation in SC $\Sigma \Delta$ modulators is the incomplete transfer of charge in the SC integrators. This nonideal effect is a consequence of the operational amplifier nonidealities, namely finite gain and BW, SR, and saturation voltages [17]. These will be considered separately in the following sections. Fig. 6 shows the model of the real integrator including all the nonidealities. A similar model, based on the same building blocks can also be realized for real resonators. 


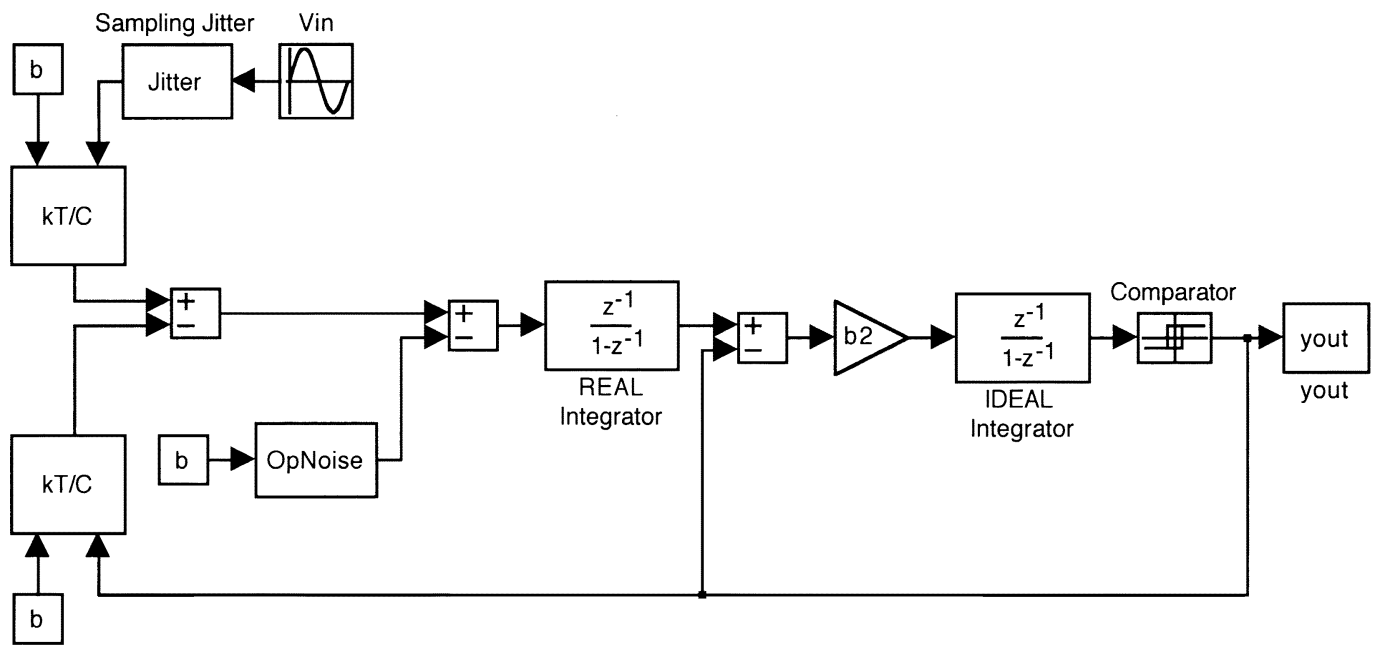

Fig. 7. Low-pass second-order $\Sigma \Delta$ modulator model.

TABLE II

Parameters of THE SECOND-ORDER Low-PASS $\Sigma \Delta$ MOdUlator Model

\begin{tabular}{l|c}
\hline \multicolumn{1}{c|}{ Parameter } & Value \\
\hline \hline Signal bandwidth & $B W=100 \mathrm{~Hz}$ \\
\hline Sampling frequency & $f_{s}=50 \mathrm{kHz}$ \\
\hline Oversampling ratio & $R=250$ \\
\hline Number of samples considered & $N=65536$ \\
\hline Integrator coefficients & $b=b_{2}=0.5$ \\
\hline
\end{tabular}

\section{A. DC Gain}

The dc gain of the integrator described by (5) is infinite. In practice, however, the actual gain is limited by circuit constraints and in particular by the operational amplifier open-loop gain $A_{0}$. The consequence of this integrator "leakage" is that only a fraction $\alpha$ of the previous output of the integrator is added to each new input sample. The limited dc gain of the integrator increases the in-band noise. The transfer function of the integrator with leakage becomes

$$
H(z)=\frac{z^{-1}}{1-\alpha z^{-1}}
$$

The dc gain of the integrator $H_{0}$, therefore, becomes

$$
H_{0}=H(1)=\frac{1}{1-\alpha} \text {. }
$$

For example, in the SC integrator shown in Fig. 1, the parameters $\alpha$ and $H_{0}$ are approximately given by

$$
\alpha \cong \frac{A_{0} C_{f}}{A_{0} C_{f}+C_{s}+C_{s R}} \text { and } H_{0}=\frac{C_{s}+C_{s R}+A_{0} C_{f}}{C_{s}+C_{c R}} .
$$

The effect of the finite open-loop dc gain on the integrator coefficient $b$ and hence on the modulator coefficients, is considered together with the operational amplifier finite BW and SR in the next paragraph (because of $A_{0}$ the actual integrator coefficient becomes $\alpha b$ ).

\section{B. $B W$ and $S R$}

The finite BW and the SR of the operational amplifier are modeled in Fig. 6 with a building block placed in front of the integrator or the resonator, which implements a MATLAB function. The effect of the finite BW and the SR are related to each other, and may be interpreted as a nonlinear gain [18]. In fact, finite BW and SR in SC circuits lead to a nonideal transient response within each clock cycle, thus producing an incomplete or inaccurate charge transfer to the output at the end of the integration period. Referring to the SC first-order $\Sigma \Delta$ modulator shown in Fig. 1, the evolution of the output node during the $n$th integration period (when $\Phi_{2}$ is on, between $n T_{s}-T_{s} / 2$ and $n T_{s}$ ) is given by

$$
v_{0}(t)=v_{0}\left(n T_{s}-T_{s}\right)+\alpha V_{s}\left(1-e^{-\frac{t}{\tau}}\right), 0<t<\frac{T_{s}}{2}
$$

where, $V_{s}=V_{\text {in }}\left(n T_{s}-T_{s} / 2\right), \alpha$ is the integrator leakage (which accounts for the operational amplifier finite gain $A_{0}$ ) and $\tau=$ $1 /(2 \pi \mathrm{GBW})$ is the time constant of the integrator and GBW is the unity gain frequency of the integrator loop-gain during the considered clock phase). The slope of this curve reaches its maximum value when $t=0$, resulting in

$$
\left.\frac{d}{d t} v_{0}(t)\right|_{\max }=\alpha \frac{V_{s}}{\tau} \text {. }
$$

We must now consider two separate cases. 


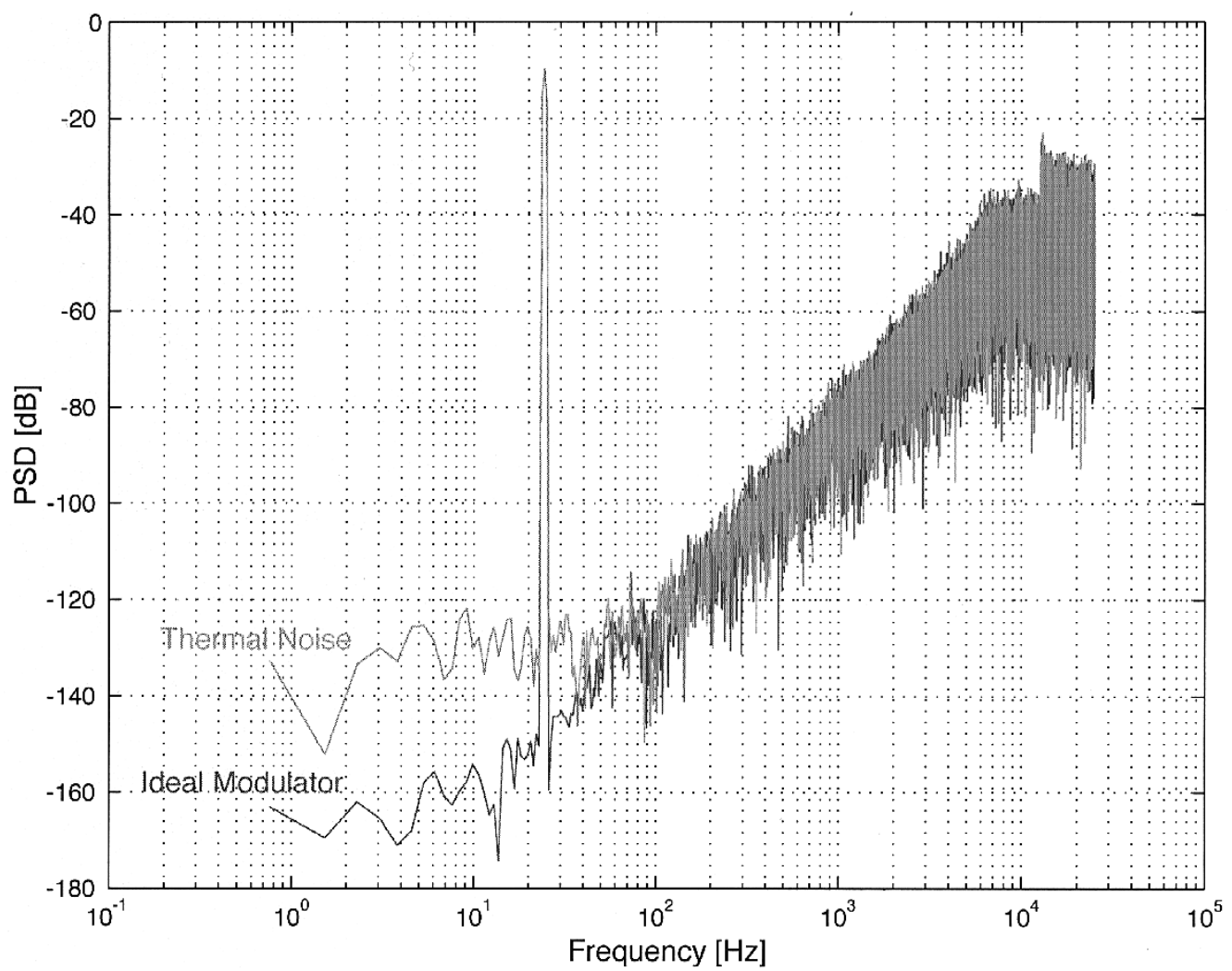

Fig. 8. PSDs of the low-pass second-order $\Sigma \Delta$ modulator output with $\left(C_{s}=2.5 \mathrm{pF}\right)$ and without (ideal modulator) the thermal noise model.

1) The value specified by (10) is lower than the operational amplifier SR (taking into account all of the capacitors connected to the operational amplifier output during the considered clock phase). In this case, no SR limitation appears and the evolution of $v_{0}$ is described by (9) during the whole clock period (until $t=T_{s} / 2$ ).

2) The value specified by (10) is larger than SR. In this case, the operational amplifier is in slewing and, therefore, the first part of the transient of $v_{0}\left(t<t_{0}\right)$ is linear with slope $S R$. The following equations hold (assuming $t_{0}<T_{s} / 2$ ):

$$
\begin{aligned}
& t \leq t_{0} \quad v_{0}(t)=v_{0}\left(n T_{s}-t_{s}\right)+S R t \\
& t>t_{0} \quad v_{0}(t)=v_{0}\left(t_{0}\right)+\left(\alpha V_{s}-S R t_{0}\right) \\
& \times\left(1-e^{-\frac{t-t_{0}}{\tau}}\right) \text {. }
\end{aligned}
$$

Imposing the condition for the continuity of the derivatives of (11)-(12) in $t_{0}$, we obtain

$$
t_{0}=\frac{\alpha V_{s}}{S R}-\tau
$$

If $t_{0} \geq T_{s} / 2$ Equation(11) holds for the whole clock period.

The MATLAB function in Fig. 6 implements the above equations to calculate the value reached by $v_{0}(t)$ at time $T_{s}$, which will be different from $V_{s}$ due to the gain, BW and SR limitations of the operational amplifier. The SR and BW limitations produce harmonic distortion reducing the total SNDR of the $\Sigma \Delta$ modulator.

\section{Saturation}

The dynamic of signals in a $\Sigma \Delta$ modulator is a major concern. It is therefore important to take into account the saturation levels of the operational amplifier used. This can simply be done in SIMULINK using the saturation block inside the feedback loop of the integrator or the resonator, as shown in Fig. 6.

\section{Simulation Results}

The proposed set of models has been used in the design of two actual circuits, namely a second-order low-pass $\Sigma \Delta$ modulator and a sixth-order bandpass $\Sigma \Delta$ modulator. Both circuits have been implemented on silicon, thus allowing the behavioral simulation results to be compared with measured data.

\section{A. Low-Pass Second-Order $\Sigma \Delta$ Modulator}

To validate the models of the various nonidealities affecting the operation of a low-pass SC $\Sigma \Delta$ modulator, we performed several simulations with SIMULINK on the second-order $\Sigma \Delta$ modulator shown in Fig. 7 [14]. Moreover, we compared the results achieved in simulation with the actual data obtained on an integrated prototype designed for microsensor applications [19]. In the circuit shown in Fig. 7 only the nonidealities of the first integrator are considered, since their effects are not attenuated by the noise shaping. This can be easily verified by using the proposed toolbox. The design parameters used for the simulations and the integrated prototype are summarized in Table II. These values correspond to the typical performance required for sensor applications. In particular, in this case a minimum SNDR of $96 \mathrm{~dB}$ (i.e. a resolution of 16 bits) is required. 


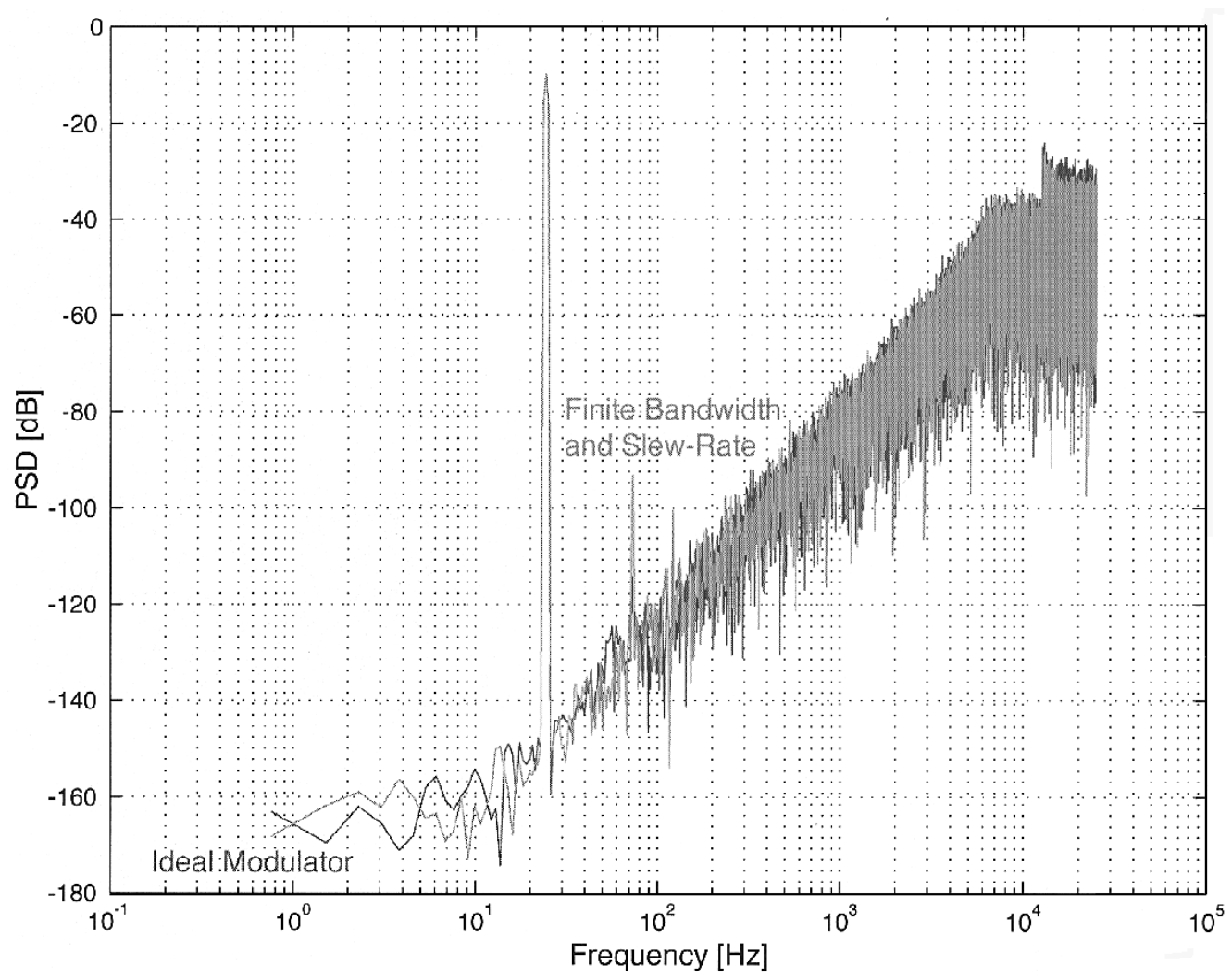

Fig. 9. PSDs of the low-pass second-order $\Sigma \Delta$ modulator output with $(S R=0.1 \mathrm{~V} / \mu$ s and GBW=100 kHz) and without (ideal modulator) the operational amplifier finite-BW and SR model.

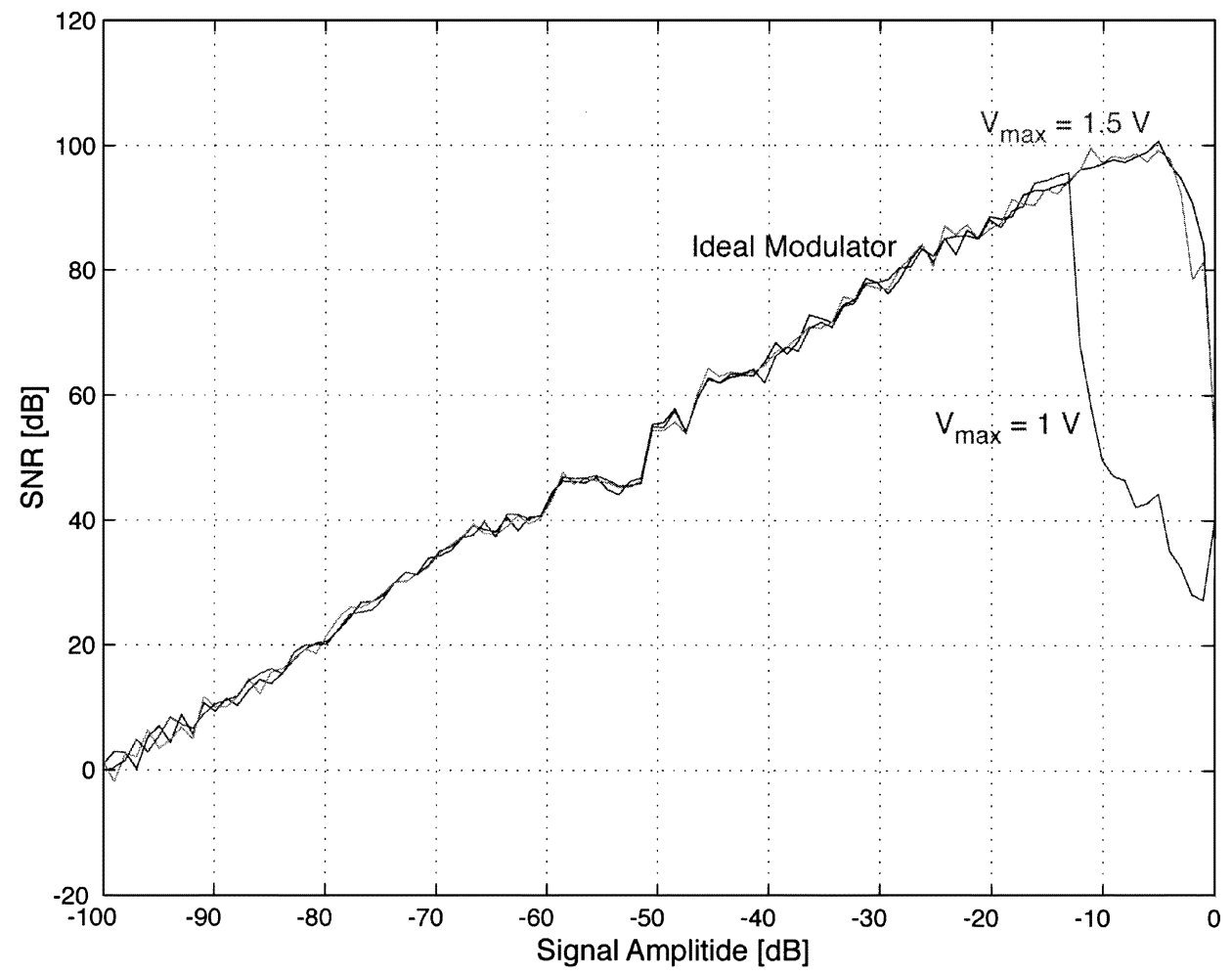

Fig. 10. SNDR of the low-pass second-order $\Sigma \Delta$ modulator as a function of the input signal amplitude for different values of the operational amplifier saturation voltage $\left(V_{\max }\right)$.

Fig. 8 shows the PSDs of the modulator output bitstream obtained in simulation without (ideal modulator) and with the thermal noise $(k T / C)$ model. The value of the sampling capacitance $C_{s}$ used in the simulation is $2.5 \mathrm{pF}$. The 
TABLE III

SNDR AND RESOLUTION OF SECOND-ORDER LOW-PASS $\Sigma \Delta$ MODULATOR

\begin{tabular}{l|c|c}
\hline \multicolumn{1}{c|}{$\Sigma \Delta$ Modulator Parameter } & $S N D R_{-6} \mathbf{d B}[d \boldsymbol{B}]$ & Resolution [bits] \\
\hline \hline Ideal modulator & 99.1 & 16.17 \\
\hline Sampling jitter $(\Delta \tau=16 \mathrm{~ns})$ & 98.8 & 16.12 \\
\hline Switches $(k T / C)$ noise $\left(C_{s}=1.25 \mathrm{pF}\right)$ & 94.0 & 15.32 \\
\hline Input-referred operational amplifier noise $\left(V_{n}=73 \mu \mathrm{V}_{\mathrm{rms}}\right)$ & 94.8 & 15.46 \\
\hline Finite dc gain $\left(H_{0}=1 \cdot 10^{3}\right)$ & 98.9 & 16.13 \\
\hline Finite bandwidth $(G B W=11.25 \mathrm{MHz})$ & 99.1 & 16.17 \\
\hline Slew-rate $(S R=4 \mathrm{~V} / \mu \mathrm{s})$ & 99.1 & 16.17 \\
\hline Saturation voltages $\left(V_{\max }= \pm 1.5 \mathrm{~V}\right)$ & 99.1 & 16.17 \\
\hline \hline Modulator simulated including all of the non-idealities & 91.6 & 14.94 \\
\hline Measurement results on the integrated prototype & 90.2 & 14.69 \\
\hline
\end{tabular}

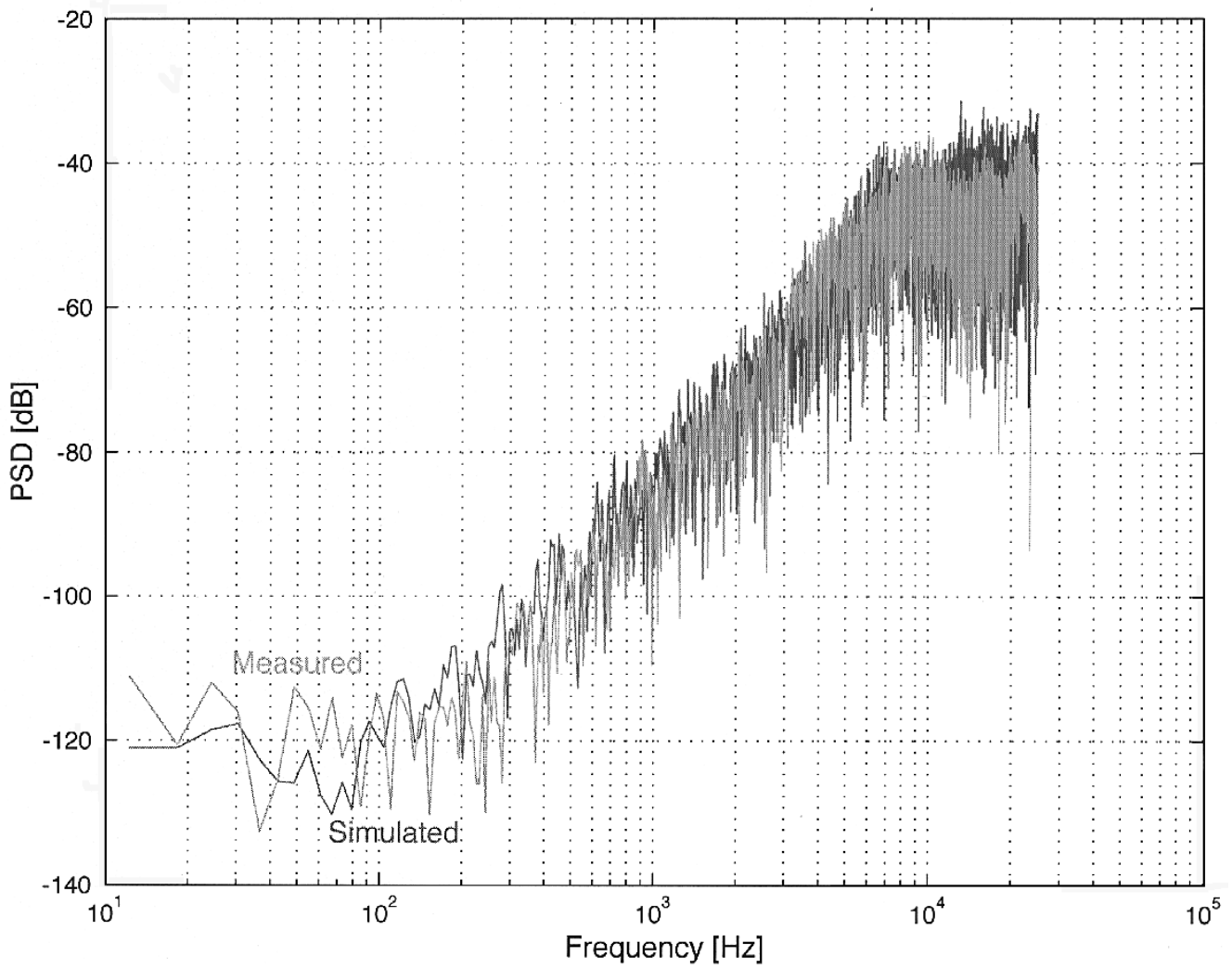

Fig. 11. Noise PSDs of the low-pass second-order $\Sigma \Delta$ modulator resulting from simulation (considering all of the nonidealities) and measurements.

$k T / C$ noise produces a noise floor at low frequencies, as expected.

Likewise, Fig. 9 shows the PSDs of the modulator output obtained in simulation without (ideal modulator) and with the operational amplifier finite BW and SR model. The SR and BW (GBW) values used in the simulation are $0.1 \mathrm{~V} / \mu$ s and $100 \mathrm{kHz}$, respectively. The finite BW and SR lead to harmonic distortion, thus degrading the SNDR performance of the $\Sigma \Delta$ modulator.

The effect of the operational amplifier saturation voltage $\left(V_{\max }\right)$ on the performance of the $\Sigma \Delta$ modulator is illustrated in Fig. 10, by plotting the simulated SNDR as a function of the input signal amplitude for different values of $V_{\max }$. The ideal modulator operates properly up to a signal amplitude of $-6 \mathrm{~dB}$, with respect to the reference voltage. A saturation voltage $V_{\max }=1.5 \mathrm{~V}$ with a reference voltage of $1 \mathrm{~V}$ does not degrade the performance significantly, while for $V_{\max }=1.0 \mathrm{~V}$ a significant degradation occurs for signal amplitudes larger than $-13 \mathrm{~dB}$, since, due to the saturation of the operational amplifiers, the modulator loop cannot follow the input signal. 
TABLE IV

Parameters of THE SiXTH-ORder BandPass $\Sigma \Delta$ Modulator Model

\begin{tabular}{l|c}
\hline \multicolumn{1}{c|}{ Parameter } & Value \\
\hline \hline Sampling frequency & $f_{s}=42.8 \mathrm{MHz}$ \\
\hline Center (intermediate) frequency & $f_{0}=10.7 \mathrm{MHz}$ \\
\hline$f_{s} / f_{0}$ ratio & 4 \\
\hline Signal bandwidth & $B W=200 \mathrm{kHz}$ \\
\hline Oversampling ratio & $R=107$ \\
\hline Number of samples considered & $N=65536$ \\
\hline
\end{tabular}

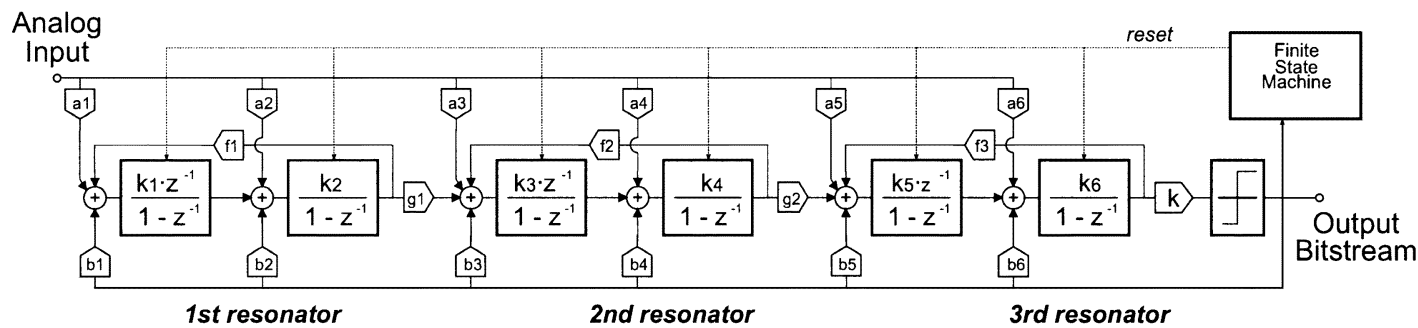

Fig. 12. Block diagram of the sixth-order bandpass $\Sigma \Delta$ modulator.

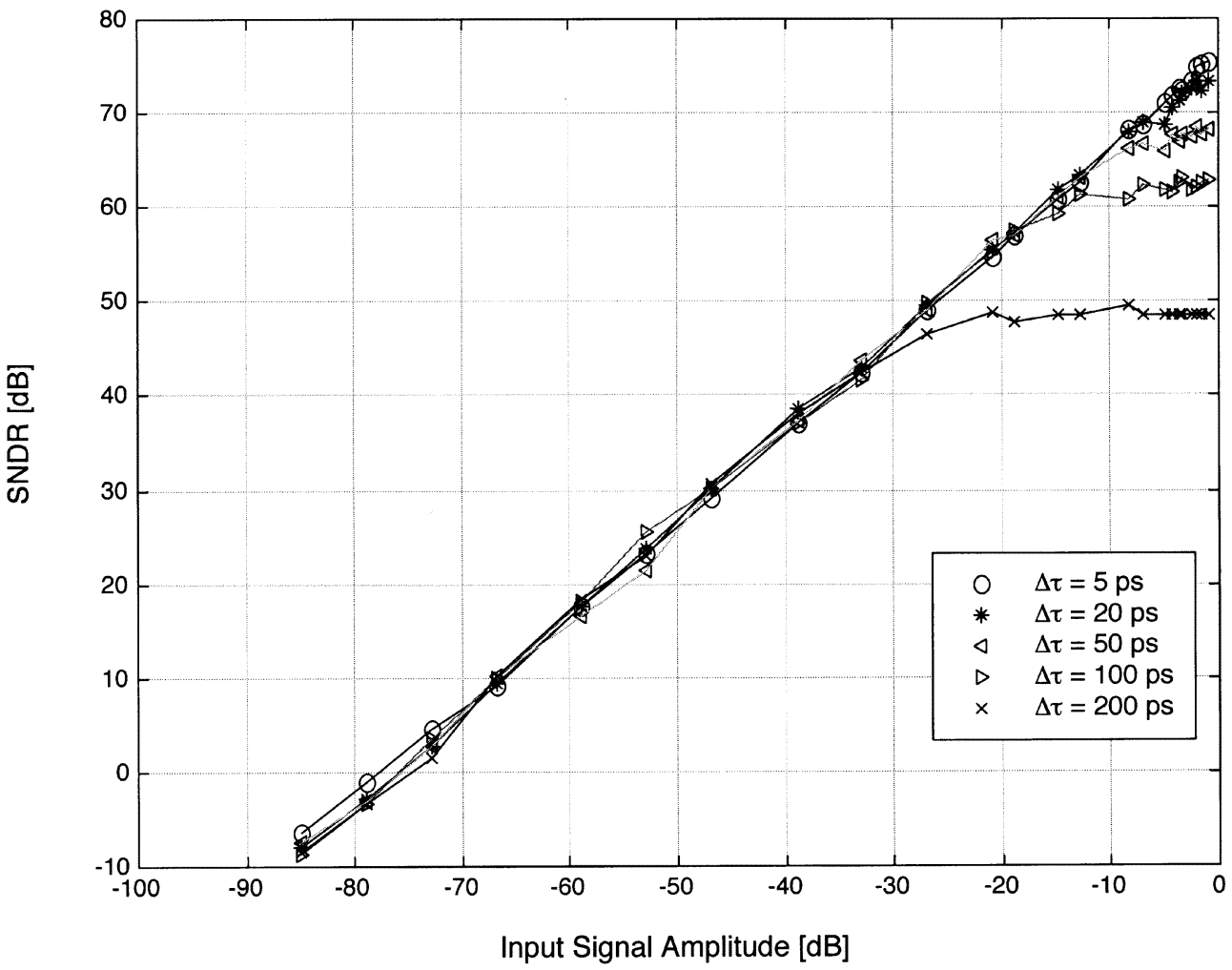

Fig. 13. SNDR as a function input amplitude for different values of the sampling jitter.

The curves shown in Fig. 8-10 show that the nonideal effects resulting from practical circuit limitations, such as thermal noise or operational amplifier nonidealities, increase the in-band noise and distortion, thus becoming potentially a severe limitation to the performance achievable with a given architecture.
Finally, Table III compares the SNDR obtained with an input signal of $-6 \mathrm{~dB}\left(\mathrm{SNDR}_{-6 \mathrm{~dB}}\right)$ with respect to full-scale and the corresponding equivalent resolution in bits of the ideal modulator, which are the maximum obtainable with the architecture and parameters used, with those achieved with the same architecture when one single nonideality at a time is introduced. 


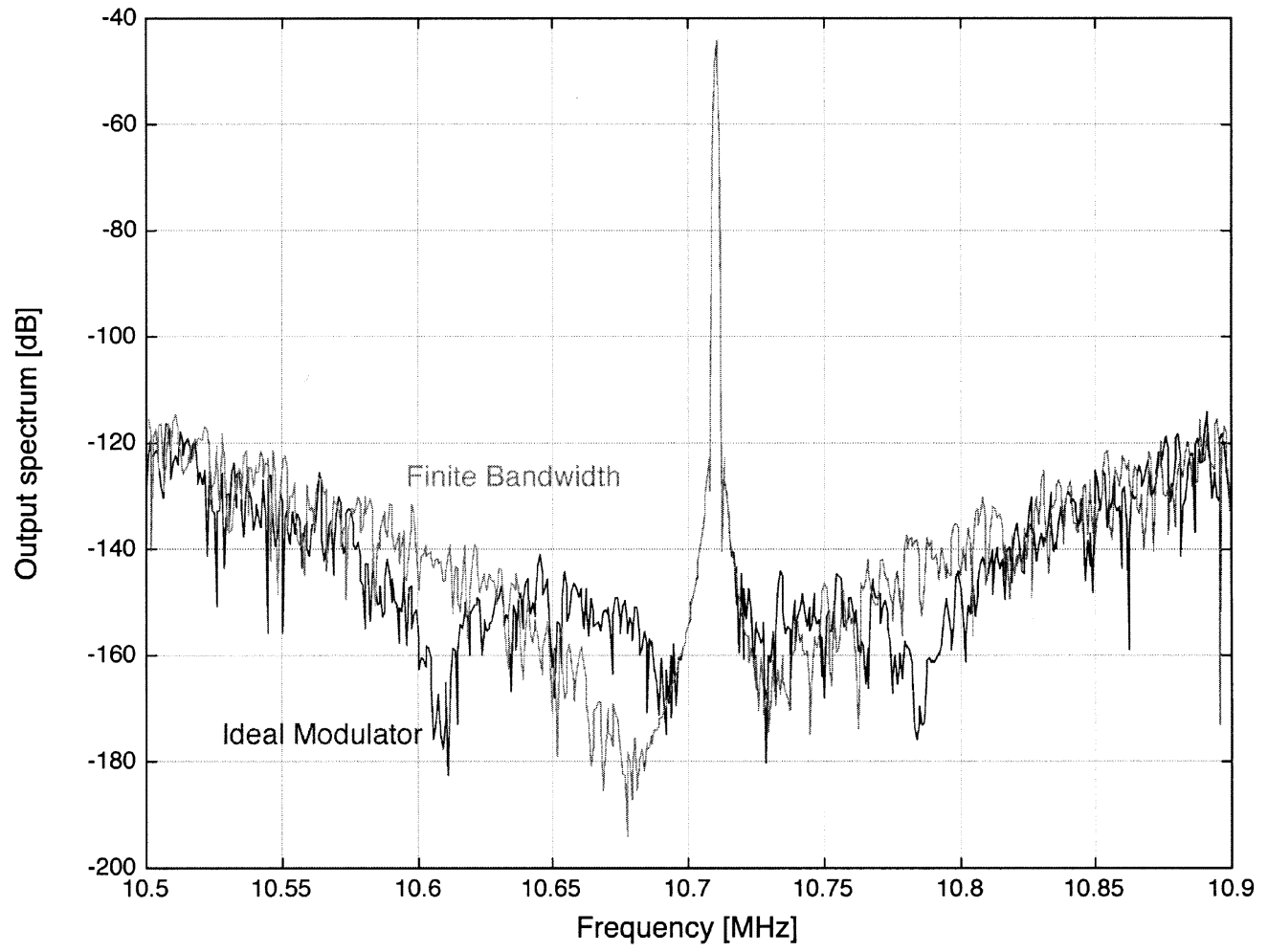

Fig. 14. PSDs of the bandpass sixth-order $\Sigma \Delta$ modulator output showing the effect of the operational amplifier finite $\mathrm{BW}(\mathrm{GBW}=150 \mathrm{MHz})$.

TABLE V

Design Parameters Used for the SiXth-Order BandPass $\Sigma \Delta$ Modulator Simulation

\begin{tabular}{l|c}
\hline \multicolumn{1}{c|}{$\Sigma \Delta$ Modulator Parameter } & Value \\
\hline \hline Sampling jitter $(\Delta \tau)$ & $20 \mathrm{ps}$ \\
\hline Finite DC gain $\left(H_{0}\right)$ & $10^{6}$ \\
\hline Finite bandwidth $(G B W)$ & $250 \mathrm{MHz}$ \\
\hline Slew-rate $(S R)$ & $300 \mathrm{~V} / \mu \mathrm{s}$ \\
\hline Saturation voltages $\left(V_{\max }\right)$ & $\pm 2 \mathrm{~V}$ \\
\hline
\end{tabular}

Moreover, the overall (SNDR_6 dB achieved in simulation considering all of the nonidealities is compared with the measured data obtained on the integrated prototype, fabricated using a double-poly, double-metal 2- $\mu \mathrm{m}$ CMOS technology. The values of the parameters used in the simulations correspond to the design parameters of the chip. Fig. 11 compares the noise PSD obtained in simulation considering all of the nonidealities with the noise PSD resulting from the measured data.

\section{B. Bandpass Sixth-Order $\Sigma \Delta$ Modulator}

To validate the models of the various nonidealities affecting the operation of a SC bandpass $\Sigma \Delta$ modulator, we performed several simulations with SIMULINK on the sixth-order modulator shown in Fig. 12 [20], [21]. In this model, the nonidealities of all the integrators were considered. The features of the bandpass modulator and the simulation parameters are reported in Table IV.
Bandpass $\Sigma \Delta$ modulators are more sensitive to sampling jitter than low-pass ones [13]. Fig. 13 shows the simulated SNDR as a function of the input amplitude for different values of the sampling jitter $(\Delta \tau)$. The limitation due to the jitter appears mainly in a limitation of the SNDR peak and not in the dynamic range (DR).

Fig. 14 shows the effect of an operational amplifier finite BW $(\mathrm{GBW}=150 \mathrm{MHz})$ on the output PSD of the SC bandpass $\Sigma \Delta$ modulator. In this case, the operational amplifier nonidealities produce a shift of the notch frequencies in the bandpass noise transfer function. However, the application requirements (and hence the decimating filter center frequency) are restricted to a given BW and therefore the shift of the notch frequencies results in an increase of the in-band quantization noise.

Fig. 15 shows the SNDR as a function of the input signal amplitude when an SR (positive and negative) of $135 \mathrm{~V} / \mu$ s and saturation voltages of $\pm 1.1 \mathrm{~V}$ are used. The effect of both these nonidealities is a degradation of the SNDR for large input signal amplitude, due to the saturation of the $\Sigma \Delta$ modulator loop. 


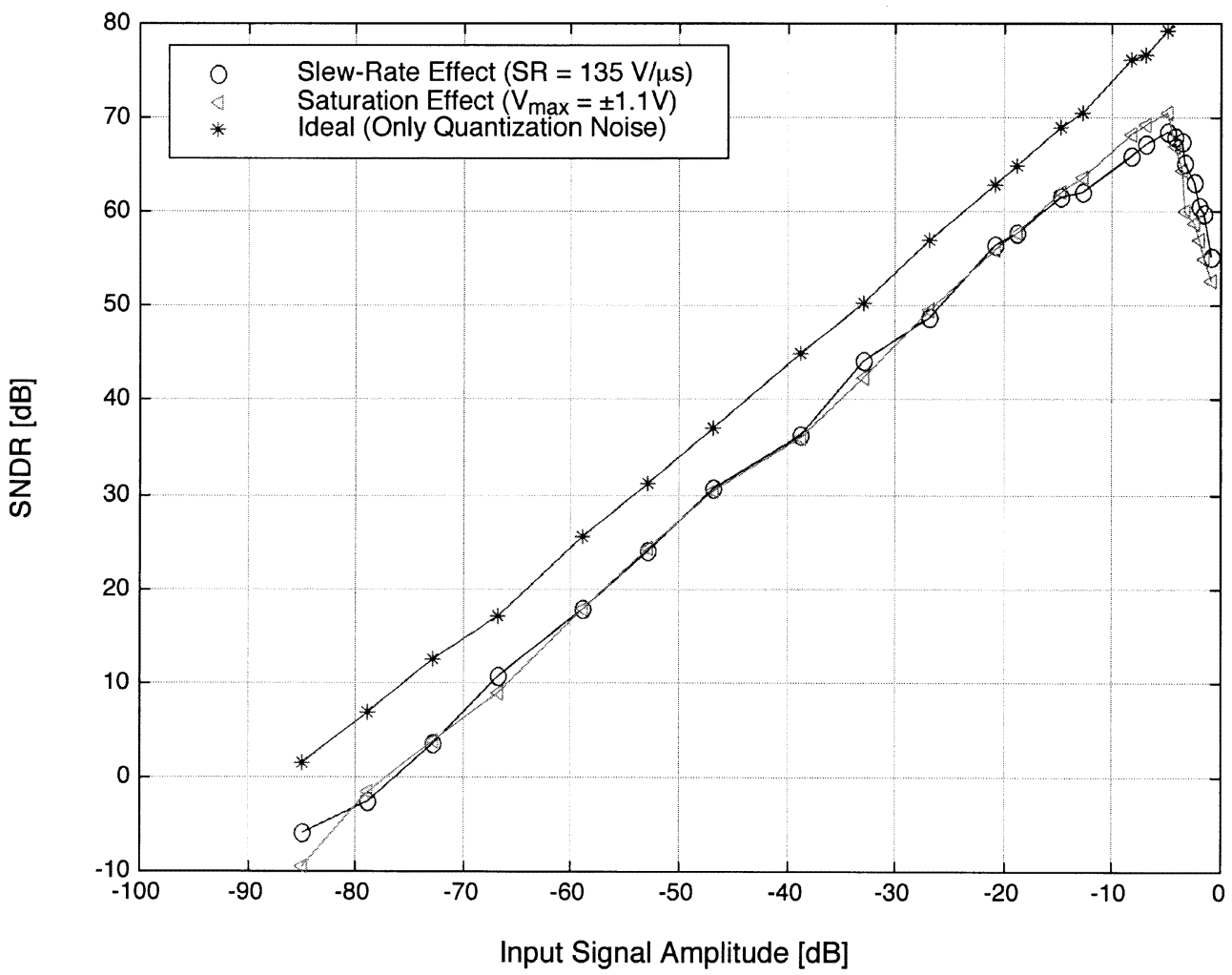

Fig. 15. SNDR as a function input amplitude with operational amplifier $\operatorname{SR}(S R=135 \mathrm{~V} / \mu \mathrm{s})$ and saturation voltage $\left(V_{\max }= \pm 1.1 \mathrm{~V}\right)$.

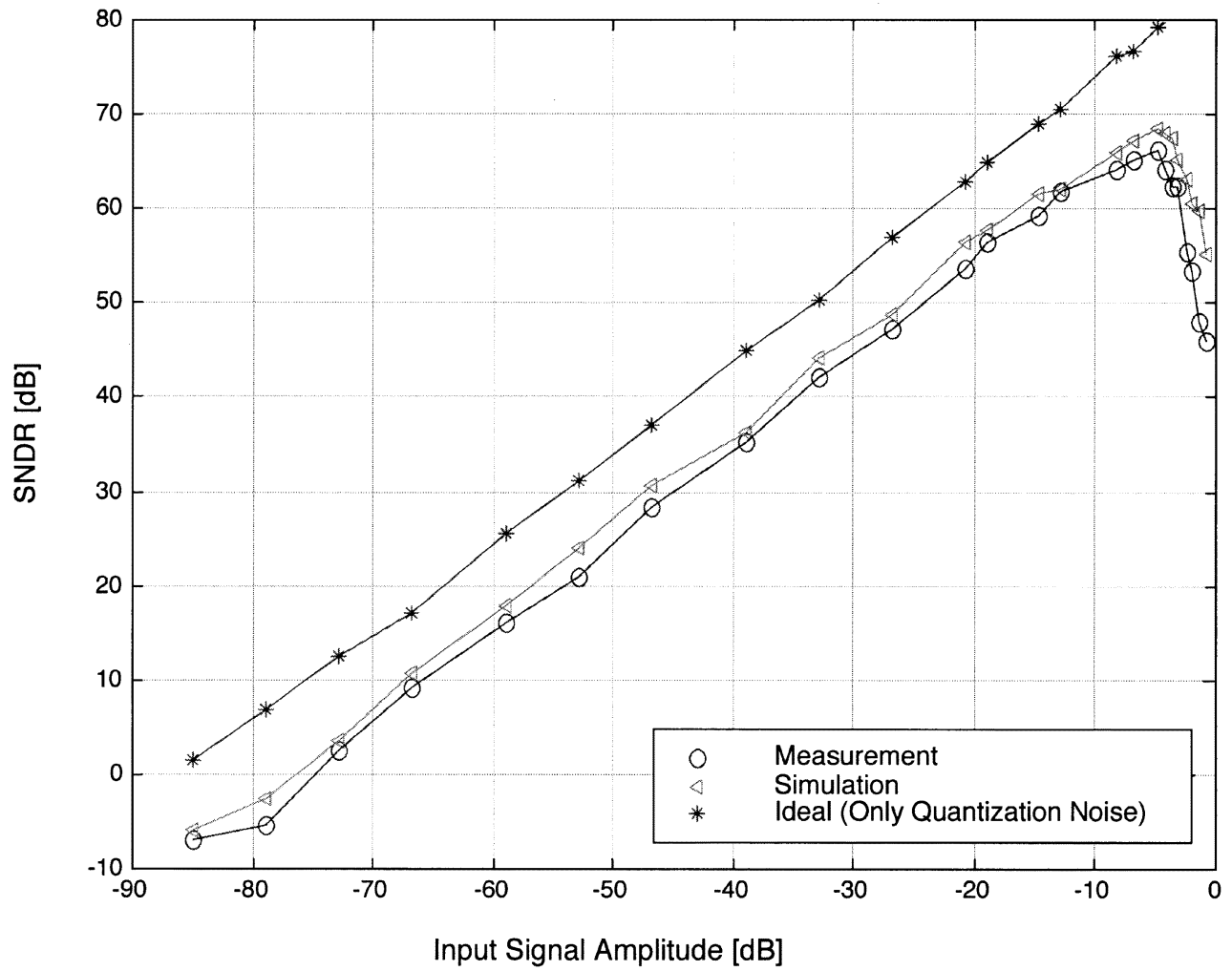

Fig. 16. SNDR as a function of the input signal amplitude of the bandpass sixth-order $\Sigma \Delta$ modulator resulting from simulation (considering all of the nonidealities) and measurements.

The modulator presented in this section has been integrated in a double-poly $0.35-\mu \mathrm{m}$ CMOS $3.3-\mathrm{V}$ technology with five metal layers. Fig. 16 compares the measured data obtained from the test chip with the simulated behavior obtained including all of the nonidealities with the parameter values reported in Table V, extracted from circuit simulation at the transistor-level. 
The measured SNDR as a function of the input signal amplitude is in good agreement with the results of the behavioral simulations. By comparing Fig. 15 and Fig. 16, it appears that operational amplifier SR and saturation are the dominant effects which affect the performance of the modulator.

\section{CONCLUSION}

In this paper, we presented a set of models implemented in the popular MATLAB SIMULINK environment suitable for timedomain behavioral simulations of SC $\Sigma \Delta$ modulators. The proposed set of models takes into account at the behavioral level most of the SC $\Sigma \Delta$ modulator nonidealities, such as sampling jitter, $k T / C$ noise, and operational amplifier parameters (white noise, finite dc-gain, finite BW, SR and saturation voltages), thus allowing us to obtain a good estimation of the $\Sigma \Delta$ modulator performance with a short simulation time (about 4096 simulated samples per second on the second-order $\Sigma \Delta$ modulator, including post-processing of the output data). The proposed simulation environment has been validated by comparing the simulated behavior with the experimental results obtained from both a second-order low-pass and a sixth-order bandpass $\Sigma \Delta$ modulator. The proposed set of models will be expanded in the future to include additional nonidealities, such as the nonlinearity of the sampling switches and of the D/A converter.

\section{APPENDIX}

The SNR and the SNDR of a $\Sigma \Delta$ modulator are defined as

$$
\mathrm{SNR}=\frac{P_{S}}{P_{N}} \text { and SNDR }=\frac{P_{S}}{P_{N}+P_{D}}=\frac{P_{S}}{P_{N+D}}
$$

respectively, where $P_{S}$ denotes the signal power, $P_{N}$ the noise power, and $P_{D}$ the power of the harmonics of the signal. In an ideal $\Sigma \Delta$ modulator, the SNR is determined only by the quantization noise according to

$$
\mathrm{SNR}=\frac{\frac{\Delta^{2}}{8}}{P_{N}}=\frac{2^{2 N} 3(2 L+1) M^{2 L+1}}{2 \pi^{2 L}}
$$

where $\Delta$ denotes the input range of the $\Sigma \Delta$ modulator, $N$ the number of bits in the quantizer, $M$ the oversampling ratio, and $L$ the order of the $\Sigma \Delta$ modulator.

However, the other noise or distortion sources increase the total noise power of the data converter above the quantization noise level and contribute to both the SNR and the SNDR.

The calculation of the SNR or SNDR of a $\Sigma \Delta$ modulator starting from the raw output data (output samples) is performed in two steps. In the first step, the sinusoidal signal $(S)$ is extracted from the sequence of $N_{O}$ output data $\left(O_{i}\right.$, at time $\left.t_{i}\right)$, typically by computing a discrete Fourier transform (DFT) of $O$ at the signal frequency $\left(f_{\text {in }}\right)$

$$
\begin{aligned}
S\left(t_{j}\right)= & \frac{1}{N_{O}}\left(\sum_{i=1}^{N_{O}} 2 O_{i} W_{i} \cos \left(2 \pi f_{\text {in }} t_{i}\right)\right) \cos \left(2 \pi f_{\text {in }} t_{j}\right) \\
& +\frac{1}{N_{O}}\left(\sum_{i=1}^{N_{O}} 2 O_{i} W_{i} \sin \left(2 \pi f_{\text {in }} t_{i}\right)\right) \sin \left(2 \pi f_{\text {in }} t_{j}\right)
\end{aligned}
$$

where $W_{i}$ denotes the desired window for the data (typically the Hanning window). The obtained signal is then subtracted from the raw output signal in the time domain, thus obtaining a signal $\left(N_{T}\right)$ which contains only the noise and distortion contributions. In the second step, we calculate the FFT of $S$ and of
$N_{T}=N+D$, obtaining the spectra of the signal $\left(S_{S}\right)$ and of the noise $\left(S_{N+D}\right)$. The same window $W_{i}$ used for the DFT has to be used also for the FFT. Finally, the signal $\left(P_{S}\right)$ and noise $\left(P_{N+D}\right)$ power are calculated by integrating the power spectra

$$
P_{S}=\sum_{i=1}^{N_{B}} S_{S}^{2}(i) \text { and } P_{N+D}=\sum_{i=1}^{N_{B}} S_{N+D}^{2}(i)
$$

where $N_{B}=N_{O} B W / f_{s}$ denotes the number of samples corresponding to the desired $\mathrm{BW}$ (baseband, BW) with sampling frequency $f_{s}$. The SNR (or SNDR) is then obtained from (14).

\section{REFERENCES}

[1] Category: Control Systems, File: SD Toolbox [Online]. Available: http://www.mathworks.com/matlabcentral/fileexchange

[2] B. P. Del Signore, D. A. Kerth, N. S. Sooch, and E. J. Swanson, "A monolithic 20-b delta-sigma A/D converter," IEEE J. Solid-State Circuits, vol. 25, pp. 1311-1317, Dec. 1990.

[3] D. A. Kerth, D. B. Kasha, T. G. Melissinos, D. S. Piasecki, and E. J. Swanson, "A 120 dB linear switched-capacitor delta-sigma modulator," in Proc. IEEE Int. Solid-State Circuit Conf. (ISSCC '94), San Francisco, CA, 1994, pp. 196-197.

[4] O. Nys and R. K. Anderson, "A 19-bit low-power multibit sigma-delta ADC based on data weighted averaging," IEEE J. Solid-State Circuits, vol. 32, pp. 933-942, July 1997.

[5] S. R. Norsworthy, R. Schreier, and G. C. Temes, "Delta-sigma data converters," in Theory, Design and Simulation. Piscataway, NJ: IEEE Press, 1997.

[6] R. Trihy and R. Rohrer, "A switched capacitor circuit simulator: AWEswit," IEEE J. Solid-State Circuits, vol. 29, pp. 217-225, Mar. 1994.

[7] G. Brauns, R. Bishop, M. Steer, J. Paulos, and S. Ardalan, "Table-based modeling of delta-sigma modulators using ZSIM," IEEE Trans. Comput. Aided Design, vol. 9, pp. 142-150, Feb. 1990.

[8] M. Lansirinne and K. Halonen, "SSDSIM-a very fast and versatile simulator for SD-modulators," in Proc. ECCTD'99, Sept. 1999, pp. 1071-1074.

[9] C. M. Wolff and L. R. Carley, "Simulation of delta-sigma modulators using behavioral models," in Proc. IEEE Int. Symp. Circuits and Systems, 1990, pp. 376-379.

[10] SIMULINK and MATLAB Users Guides, The MathWorks, Inc., Natick, MA, 1997.

[11] SigDel, R. Schreier. [Online]. Available: http://www.mathworks.com/ matlabcentral/fileexchange/index.jsp

[12] E. O. Bringham, The Fast Fourier Transform. Englewood Cliffs, NJ: Prentice-Hall, 1974

[13] H. Hai Tao, L. Toth, and J. M. Khoury, "Analysis of timing jitter in bandpass sigma-delta modulators," IEEE Trans. Circuits Syst. II, vol. 46, pp. 991-1001, Aug. 1999.

[14] B. E. Boser and B. A. Wooley, "The design of sigma-delta modulation analog-to-digital converters," IEEE J. Solid-State Circuits, vol. 23, pp. 1298-1308, Dec. 1988.

[15] V. F. Dias, G. Palmisano, and F. Maloberti, "Noise in mixed continuous-time switched-capacitor sigma-delta modulators," Proc. Inst Elect. Eng. G, vol. 139, pp. 680-684, Dec. 1992.

[16] C. C. Enz and G. C. Temes, "Circuit techniques for reducing the effects of op-amp imperfections: autozeroing, correlated double sampling, and chopper stabilization," Proc. IEEE, vol. 84, pp. 584-1614, Nov. 1996.

[17] G. Temes, "Finite amplifier gain and bandwidth effects in switched-capacitor filters," IEEE J. Solid-State Circuits, vol. 15, pp. 358-361, June 1980.

[18] F. Medeiro, B. Perez-Verdu, A. Rodriguez-Vazquez, and J. L. Huertas, "Modeling opamp-induced harmonic distortion for switched-capacitor $\Sigma \Delta$ modulator design," in Proc. IEEE Int. Symp. Circuits and Systems (ISCAS '94), vol. 5, London, U.K., May 1994, pp. 445-448.

[19] P. Malcovati, A. Häberli, F. Mayer, O. Paul, F. Maloberti, and H. Baltes, "Combined air humidity and flow CMOS microsensor with on-chip 15 bit sigma-delta A/D interface," in Dig. IEEE VLSI Circuit Symp. (VLSI '95), Kyoto, Japan, Jun. 1995, pp. 45-46.

[20] D. Tonietto, P. Cusinato, F. Stefani, and A. Baschirotto, "A 3.3 V CMOS 10.7 MHz 6th-order bandpass sigma-delta modulator with $78 \mathrm{~dB}$ dynamic range," in Proc. IEEE Eur. Solid-State Circuit Conf. (ESSCIRC '99), Duisburg, Germany, Sep. 1999, pp. 78-81.

[21] D. Tonietto, P. Cusinato, F. Stefani, and A. Baschirotto, "Considerations for the design of a $10.7 \mathrm{MHz}$ band-pass sigma-delta modulator," in Proc. Eur. Conf. Circuit Theory and Design (ECCTD '99), Stresa, Italy, Aug. 1999, pp. 5-8. 


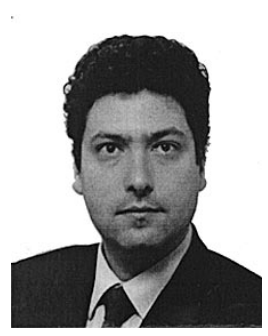

Piero Malcovati (S'94-M'95) was born in Milan, Italy in 1968. He received the Laurea degree (summa cum laude) in electronic engineering from the University of Pavia, Pavia, Italy, in 1991, and the Ph.D. degree in electrical engineering from The Swiss Federal Institute of Technology (ETH), Zurich, Switzerland, in 1996.

From 1996 to 2001, he was an Assistant Professor in the Department of Electrical Engineering, the University of Pavia, where since 2002, he has been an Associate Professor of Electrical Measurement. His research activities are focused on microsensor interfaces and high performance data converters. He has authored or coauthored more than 20 papers in international journals, more than 60 presentations at international conferences (with published proceedings), two book chapters, and holds three industrial patents.

Dr. Malcovati has been Special Session Chairman of IEEE ICECS 2001 and Technical Program Committee Secretary of ESSCIRC 2002. He has been Guest Editor of the Journal of Analog Integrated and Signal Processing for the special issue on IEEE ICECS 1999. In 1991, he received a one-year grant from SGSThomson, Italy.

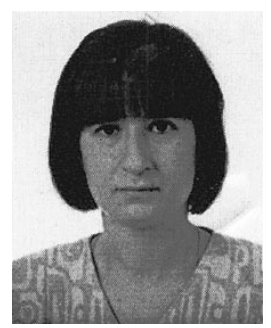

Simona Brigati (S'89-M'96) was born in Castelsangiovanni (PC), Italy, in 1966. She received the graduate degree (summa cum laude) in electronic engineering, and the Ph.D. degree in electronic and computer engineering (working on the design of high-speed A/D and D/A converters), from the University of Pavia, Pavia, Italy, in 1991 and 1995, respectively.

From 1996 to 2000, she worked for Micronova Sistemi S.r.1., Pavia, Italy, and in 2001, she joined ACCO Microelettronica S.r.1, Pavia, Italy, where she is involved in the design of mixed analog-digital ASICs. Her research interests are in the field of high-speed CMOS data converters, high resolution sigma-delta modulators and high-performance analog and mixed ICs.

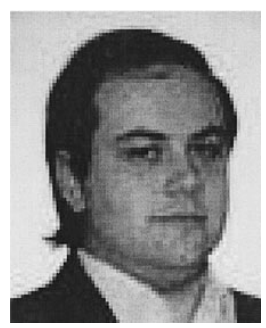

Fabrizio Francesconi (S'95-M'00) was born in Mortara (PV), Italy, in 1965 . He received the Laurea degree in electronics and the Ph.D. degree in electronic and computer engineering (working on the design of bandpass sigma-delta A/D converters), from the University of Pavia, Pavia, Italy, in 1991, and 1996, respectively.

From 1996 to 2000, he worked for Micronova Sistemi S.r.l., Pavia, Italy, and in 2001, he joined ACCO Microelettronica S.r.l, Pavia, Italy, where he is responsible for the development of mixed analog-digital integrated circuits. His main interests are in the area of audio integrated circuits, SC filters, A/D and D/A converters, and sigma-delta converters.

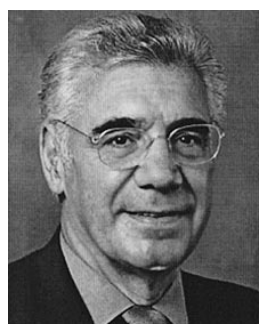

Franco Maloberti (SM'87-F'96) received the Laurea degree in physics (summa cum laude) from the University of Parma, Parma, Italy, in 1968, and the Dr. Honoris Causa Ph.D. in electronics from the Instituto Nacional de Astrofisica, Optica y Electronica (Inaoe), Puebla, Mexico, in 1996.

In 1993, he was a Visiting Professor at The Swiss Federal Institute of Technology (ETH-PEL), Zurich, Switzerland. He was Professor of Microelectronics and Head of the Micro Integrated Systems Group, University of Pavia, Italy and the TI/J.Kilby Analog Engineering Chair Professor at the A\&M University, Houston, TX. He is currently the Distinguished Microelectronic Chair Professor at the University of Texas at Dallas and part-time Professor at the University of Pavia, Pavia, Italy. His professional expertise is in the design, analysis, and characterization of integrated circuits and analog digital applications, mainly in the areas of switched-capacitor circuits, data converters, interfaces for telecommunication and sensor systems, and CAD for analog and mixed A/D design. He has written more than 250 published papers, three books, and holds 15 patents. He has been responsible at both technical and management levels for many research programs including 10 ES-PRIT projects and has served the European Commission as ESPRIT Projects' Evaluator, Reviewer and as European Union expert in many European Initiatives.

Dr. Maloberti was the recipient of the XII Pedriali Prize for his technical and scientific contributions to national industrial production, in 1992. He was co-recipient of the 1996 Institute of Electrical Engineers (U.K.) Fleming Premium for the paper "CMOS Triode Transistor Transconductor for high-frequency continuous time filters." He served the Academy of Finland and the Portuguese Research Council in the assessment of electronic research in Academic institutions and on the research programs' evaluations. He is the President of the IEEE Sensor Council. He was Vice-President, Region 8, of the IEEE Circuits and Systems Society from 1995 to 1997 and an Associate Editor of IEEE TRANSACTIONS ON CIRCUITS AND SYSTEMS-II. He received the 1999 IEEE CAS Society Meritorious Service Award, the 2000 CAS Society Golden Jubilee Medal, and the 2000 IEEE Millennium Medal. He is a member of the Italian Electrotecnical and Electronic Society (AEI), the Editorial Board of Analog Integrated Circuits and Signal Processing.

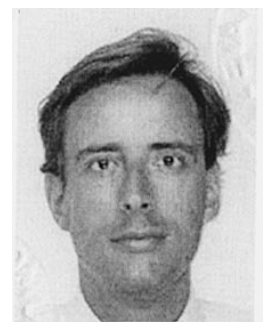

Paolo Cusinato was born in Lavagna, Italy, in 1969. He received the degree of Ingegnere (summa cum laude) from the University of Genoa, Genoa, Italy, in 1993, with a dissertation on the subject of high-speed A/D conversion.

In 1994, he was with the Centre Suisse d'Electronique et de Microtechnique (CSEM), Neuchatel, Switzerland, where he was involved in the design of low-power ICs for image filtering. From 1995 to 1999 , he was with ST Microelectronics, Milan, Italy, working on digital radio systems, front ends for CD applications and digital signal processing. Currently, he is with Texas Instruments, Villeneuve-Loubet, France, working on analog baseband signal processing for wireless applications (GSM/WCDMA). His main interest is in CMOS mixed analog/digital ICs, in particular, A/D and D/A converters, and high-speed PLLs. Dr. Cusinato has published more than ten technical papers and received 15 patents in this area.

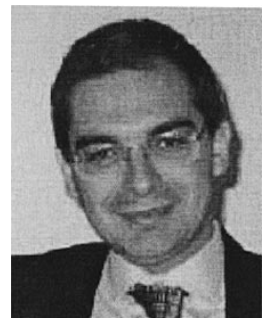

Andrea Baschirotto (S'89-M'95-SM'01) was born in Legnago (Verona), Italy, in 1965. He received the graduate degree in electronic engineering (summa cum laude) and the Ph.D. degree in electrical engineering, from the University of Pavia, Pavia, Italy, in 1989, and 1994, respectively.

In 1994, he joined the Department of Electronics, University of Pavia, as a Researcher (Assistant Professor). In 1998, he joined the Department of Innovation Engineering, University of Lecce, Lecce, Italy, as an Associate Professor. Since 1989, he has collaborated with STMicroelectronics, Cornaredo, Italy, on the design of ASICs. Since 1991, he has been associated with I.N.F.N. (1991-1998 with Section of Milan and in 1999 with Section of Lecce) on the design and realization of readout channels for high-energy physics experiments (such as L3) and space experiments (such as AMS). In 1999/2000, he collaborated with Medico S.p.A., Rubano, Italy, on the design of a low-power front end for implantable device (pacemaker) applications. His main research interests are in the design of mixed analog/digital integrated circuits, in particular for low-power and/or high-speed signal processing. He has authored or coauthored more than 40 papers in international journals, more than 50 presentations at international conferences (with published proceedings), two book chapters, and holds ten industrial patents. In addition, he has coauthored more than 120 papers within research collaborations on high-energy physics experiments.

Dr. Baschirotto was a Guest Editor for the IEEE TRANSACTIONS ON CIRCUITS AND SYSTEMS-PART II for the special issue on IEEE ISCAS 1998, and he is now serving IEEE TRANSACTIONS ON CIRCUITS AND SYSTEMS-PART II as an Associate Editor. He has been the Technical Program Committee Chairman for ESSCIRC 2002. 